\title{
Mechanical stretch-induced osteogenic differentiation of human jaw bone marrow mesenchymal stem cells (hJBMMSCs) via inhibition of the NF-KB pathway
}

Xiaoyan Chen', Yuan Liư', Wanghui Ding ${ }^{1}$, Jiejun Shi', Shenglai Li ${ }^{3}$, Yali Liư ${ }^{4}$, Mengjie Wư ${ }^{1}$ and Huiming Wang

\begin{abstract}
Severe malocclusion can contribute to several serious dental and physical conditions, such as digestive difficulties, periodontal disease, and severe tooth decay. Orthodontic treatment is mainly used to treat malocclusion. Forces in orthodontic tooth results in bone resorption on the pressure side and bone deposition on the tension side. Osteoblasts have been considered as the key component in bone regeneration on the tension side. However, the underlying mechanisms remain unclear. In this study, we focus on how mechanical stretch regulates the osteogenesis during orthodontic treatment. Human jaw bone marrow mesenchymal stem cells (hJBMMSCs) were isolated from healthy adult donors and cultured in regular medium (control) or osteogenic medium (OS). Under OS culture, hJBMMSCs presented osteogenic differentiation potentials, as evidenced by increased mineralization, enhanced calcium deposition, and upregulated expression of osteogenesis markers (ALP, osterix, and RunX). What's more, the OSinduced osteogenesis of hJBMMSCS is associated with the dephosphorylation of IKK, activation of IKBa, and phosphorylation/nucleic accumulation of P65, which all indicated the inhibition of NF-KB activity. Overexpressing P65 in hJBMMSCs, which could constantly activate NF-KB, prevented the osteogenic differentiation in the OS. After that, we applied the Flexcell tension system, which could cause mechanical stretch on cultured hJBMMSCs to mimic the tension forces during tooth movement. Mechanical stretch resulted in 3.5-fold increase of ALP activity and 2.4-fold increase of calcium deposition after 7 days and 21 days treatment, respectively. The expression levels of ALP, RunX2, and Osterix were also significantly upregulated. In the meantime, applying mechanical stretch on OS-cultured hJBMMSCs also dramatically promoted the OS-induced osteogenesis. Both OS and mechanical stretch downregulated NF-KB activity. By overexpressing P65 in hJBMMSCs, neither OS nor mechanical stretch could induce their osteogenesis. These results indicated that, like OS induction, mechanical stretch-facilitated osteogenesis of hJBMMSCs by inhibiting $\mathrm{NF}-\mathrm{KB}$ in the noninflammatory environments.
\end{abstract}

Correspondence: Huiming Wang (hmwang1960@hotmail.com) ${ }^{1}$ Department of Orthodontics, Affiliated Hospital of Stomatology, Medical College, Zhejiang University, Hangzhou, Zhejiang Province, China

${ }^{2}$ Department of Liver Surgery, Ren Ji Hospital Affiliated to Shanghai Jiao Tong University School of Medicine, Shanghai, China

Full list of author information is available at the end of the article

These authors contributed equally: Xiaoyan Chen, Yuan Liu.

Edited by Y. Shi

\section{Introduction}

Orthodontic tooth movement is possible due to the remodeling ability of the surrounding bone and soft tissues. Orthodontic appliances are built to generate biomechanical force systems that produce the desired tooth and jaw movements needed to establish an ideal occlu$\operatorname{sion}^{1}$. In orthodontic tooth movement, bone resorption occurs on the pressure side and bone deposition takes 
place on the tension side ${ }^{2}$. Osteoblasts have been considered as the key component in bone regeneration on the tension side ${ }^{3}$. They are derived from mesenchymal stem cells (MSCs) in the bone marrow and make up the organic bone matrix ${ }^{4}$. Osteoblasts and their precursor cells are sensitive to mechanical stimuli and have an ability to translate the mechanical signals into biological respon$\mathrm{ses}^{5}$. Various biological factors such as prostaglandin E2 (PGE), osteoprotegerin (OPG), bone morphogenetic proteins (BMPs), and nuclear factor- $\mathrm{\kappa B}(\mathrm{NF}-\mathrm{\kappa B})$ have been established as important elements in osteogenic differentiation and bone remodeling ${ }^{5,6}$.

The transcription factor NF- $\mathrm{KB}$ represents a family of five mammalian proteins (c-rel, relA, p65, NF-kB1, and NF- $\mathrm{kB} 2$ ) that regulate a myriad of genes involved in immune and inflammatory responses, among many other functions ${ }^{7}$. NF- $\mathrm{kB}$ dimers reside in cytoplasm in an inactive state through interactions with $\mathrm{\kappa B}$ inhibitors

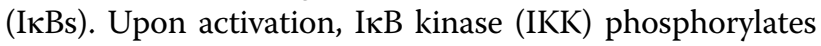
I $\kappa \mathrm{B}$ and facilitates its degradation. Dissociated NF- $\kappa \mathrm{B}$ homodimers or hetertodimers then enter the nuclei and activate transcription $^{8,9}$. Pro-inflammatory cytokines such as TNF and IL-17 can stimulate IKK-NF- $\mathrm{KB}$ and inhibit osteogenic differentiation of MSCs in inflammatory settings $^{8}$. Activation of NF- $\mathrm{kB}$ blocks c-Jun N-terminal kinase (JNK) activity and other osteoblast proteins, such as MGP, Smad and Fra1, which eventually impedes osteogenic differentiation of $\mathrm{MSCs}^{5,10,11}$. However, with respect to orthodontic tooth movement in noninflammatory environments, how mechanical signals affect NF- $\kappa \mathrm{B}$ and regulate MSCs behavior remains unknown.

Like MSCs from other sites, such as the ilium and sternum, human jaw bone marrow MSCs (hJBMMSCs) have the potential to differentiate into osteoblasts ${ }^{12,13}$. Our previous study has revealed that inflammatory microenvironments impaired osteogenesis of human periodontal ligament tissue-derived MSCs by activating GSK- $3 \beta$ and NF- $\mathrm{KB}^{4}$. In the current study, we focus on how the mechanical stretch in non-inflammatory environments regulate the osteogenesis of hJBMMSCs. We isolated hJBMMSCs from healthy adult human donors and identified their stem cell properties. In vitro mechanical stretch and osteogenic medium (OS) both could facilitate osteogenesis of hJBMMSCs by inhibiting the NF- $\mathrm{kB}$ pathway. However, overexpression of P65 constantly activated NF- $\mathrm{KB}$ and diminished the mechanical stretch or OS-induced osteogenic differentiation.

\section{Results}

\section{Characterization of isolated hJBMMSCs}

Isolated hJBMMSCs were cultured in vitro and stained with stem cell-specific markers CD29 and $\mathrm{CD} 13^{14}$. Immunofluorescence staining and flow cytometry analysis indicated high expression of CD29 and CD13 in >90\% of the cultured hJBMMSCs, while low expression of CD45 and CD146 (Fig. 1a and Supplementary Fig. 1A). Lipidforming medium treatment for 7 days in vitro resulted in lipid restoration in hJBMMSCs (Fig. 1b). Consistently, OS-cultured (osteogenic medium) hJBMMSCs indicated osteoblast characterization as evidenced by increased alkaline phosphatase activity (ALP, Fig. 1c, d) and intense alizarin red staining (Fig. 1e, f). ALP protein level increased 1.5-fold after 7 days incubation in OS (Fig. 1g). Osteoblast-specific transcription factors, Runx2 and Osterix, were also upregulated after OS induction (Fig. 1h, i). This result indicated that in vitro hJBMMSCs had stem cell properties and OS facilitated their osteogenic differentiation.

\section{Osteogenic differentiation of $\mathrm{hJBMMSC}$ inhibited NF-KB activity}

Next, we tested how NF- $\mathrm{KB}$ activity was regulated during the osteogenic differentiation of hJBMMSCs. Sixty minutes after OS induction, IKB $\alpha$ degradation was inhibited as p-IKK decreased and IKB $\alpha$ increased (Fig. 2a). Meanwhile, activity of P65 was downregulated after OS induction, as its phosphorylation and accumulation in nuclei were all inhibited (Fig. 2b, c and Supplementary Fig. 1B). This finding indicated that OS induction blocked the NF- $\mathrm{KB}$ activity of hJBMMSCs in a time-dependent manner.

\section{Activation of NF-KB blocked osteogenic differentiation}

Since osteogenic differentiation inhibited NF- $\mathrm{KB}$ activity, we tried to ascertain how activated NF- $\mathrm{KB}$ affected the osteogenesis of hJBMMSCs. Plasmids consistently overexpressing P65 were transfected into hJBMMSCs and the transfection efficiency was confirmed by quantitative reverse transcription PCR (qRT-PCR) and western blotting (Fig. 3a, b). Compared with the control group, hJBMMSCs overexpressing P65 demonstrated reduced osteogenesis after OS induction, as evidenced by less ALP activity (Fig. 3c, d) and mineralization (Fig. 3d, e). ALP expression was diminished by overexpressed P65, as indicated by western blotting and qRT-PCR (Fig. 3f, h). Meanwhile, Runx2 and Osterix were also blocked in P65pretreated hJBMMSCs (Fig. 3g, i, j). This result indicated that constant activation of NF- $\mathrm{kB}$ by overexpressing P65 blocked osteogenic differentiation of hJBMMSCs.

\section{Mechanical stretch-facilitated osteogenesis of hJBMMSCs in vitro via diminishing NF-KB activity}

The Flexcell tension system could induce mechanical stretch in vitro on cultured hJBMMSCs, which mimicked the scenario of tension stress during orthodontic tooth movement. We applied the Flexcell tension system on hJBMMSCs and found an osteogenic differentiation 


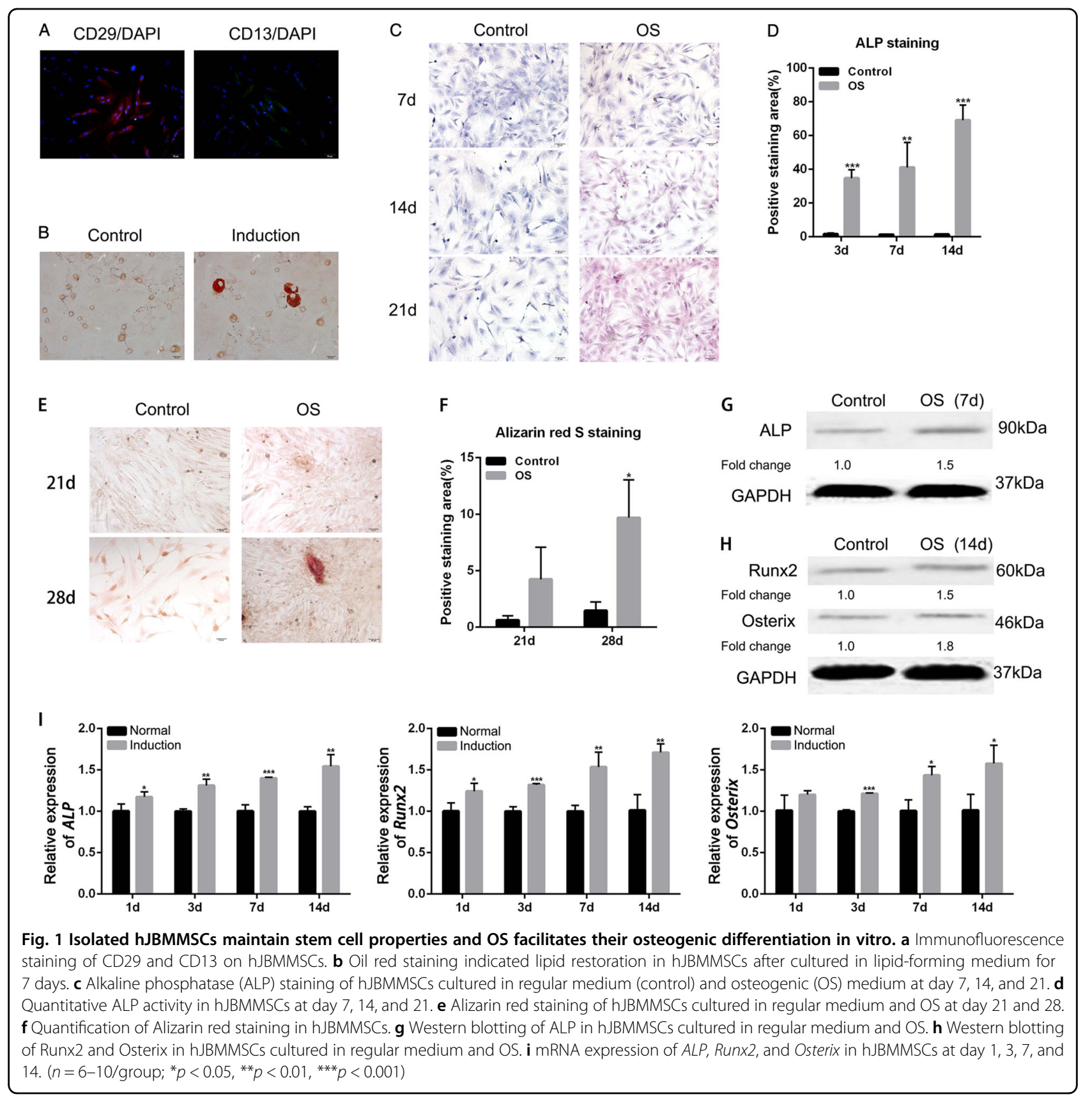

tendency, as indicated by massive positive staining of ALP (Fig. 4a, b) and mineralization (Fig. 4a, c). Meanwhile, Flexcell could facilitate the OS-induced osteogenesis of hJBMMSCs, which is accompanied with increased expression of Run $\times 2$ and Osterix (Fig. 4e, f, Supplementary Fig. 2A, B). Like how OS-induced osteogenesis could inhibit NF- $\mathrm{BB}$ activity, Flexcell-related osteogenic differentiation also eliminated P65 from nuclei (Fig. 4g and Supplementary Fig. 1C) and was positively correlated with downregulation of P65 in a time-dependent manner (Fig. 4h). IKB $\alpha$ degradation was inhibited by both Flexcell and OS (Fig. 4i). In addition, we also tested the expression of pro-inflammatory cytokines TNF- $\alpha$, IL-17, and IL-11, which were regulated by NF- $\mathrm{kB}$. qRT-PCR and ELISA revealed decreased expression and release of those cytokines after Flexcell and OS treatment (Fig. 4j-l, Supplementary Fig. 3). Thus, it can be inferred that mechanical stretch-induced osteogenesis via inhibition of $\mathrm{NF}-\kappa \mathrm{B}$ activity.

\section{Activation of NF-KB reversed mechanical stretch-mediated osteogenesis}

To further study the role of NF- $\mathrm{BB}$ in mechanical stretch-induced osteogenesis, we constantly overexpressed 


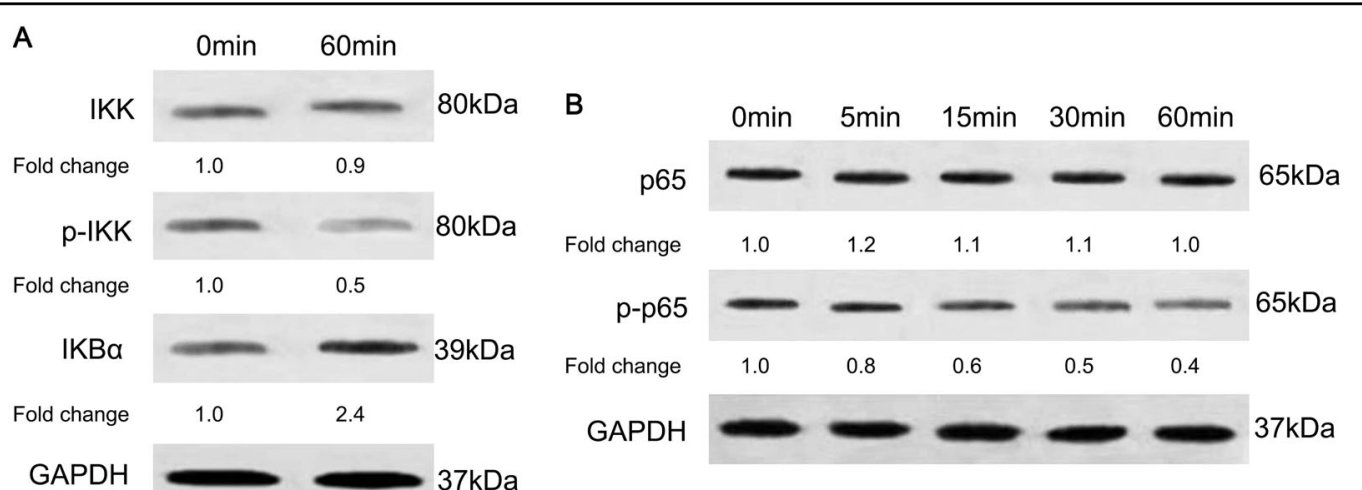

C
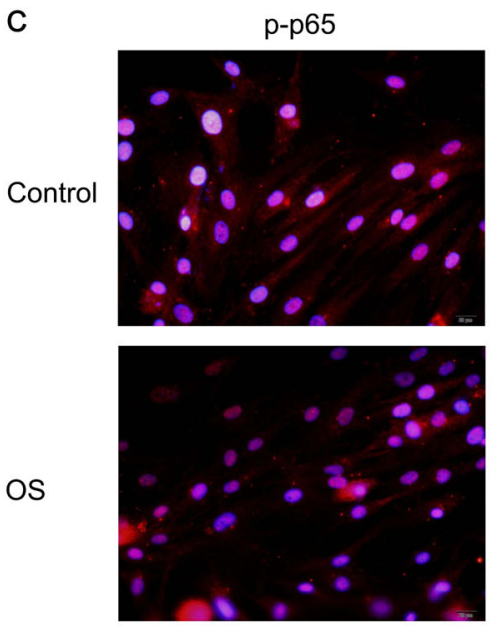

Fig. 2 OS induces osteogenic differentiation of hJBMMSCs by inhibiting NF-kB activity. a Western blotting indicated decreased phosphorylation of IKK and increased IKBa in hJBMMSCs after 60 min induction by OS. b Phosphorylation of P65 decreased in hJBMMSCs in timedependent manner after OS induction. c Immunofluorescence staining of phosphorylated P65 (p-P65) in hJBMMSCs. Less nucleic accumulation of pP65 was demonstrated after OS induction

P65 in hJBMMSCs and employed the Flexcell tension system. After Flexcell and OS treatment, lighter staining of ALP and reduced mineralization were observed in P65 overexpressed hJBMMSCs, when compared with the control group (Fig. 5a-c). Intracellular ALP level decreased $25 \%$ by over-activated P65 in the setting of Flexcell treatment (Fig. 5d). Osteoblast-related transcription factors Run $\times 2$ and Osterix were also inhibited by activated P65 (Fig. 5e). Therefore, mechanical stretch-induced osteogenic differentiation of hJBMMSCs was diminished by unlimited activation of NF-kB.

\section{Discussion}

Orthodontic tooth movement is a result of bone remodeling by differentially generating osteoblasts and osteoclasts in the pressure and tension areas around teeth. However, how mechanical stress during orthodontic tooth movement regulates osteoblast differentiation is still unknown. In this study, we isolated hJBMMSCs from healthy adult human donors and confirmed their potential for osteogenesis in osteogenic medium. The osteogenic differentiation of hJBMMSCs in vitro was achieved by inhibiting NF- $\mathrm{KB}$ activity. Meanwhile, using the Flexcell tension system to apply mechanical stretch on hJBMMSCs also facilitated their osteogenesis. NF- $\kappa B$ activity was blocked in the mechanical stretch-induced osteogenic differentiation, while constant activation of NF- $\mathrm{KB}$ diminished the differentiation. Our study is the first to confirm the osteogenic role of hJBMMSCs during mechanical stretch, which is dependent on inhibition of NF-kB.

hJBMMSCs are postnatal multipotent stem cells residing in the maxilla and mandible ${ }^{13}$. The osteoclasts and osteoblasts that present during orthodontic tooth movement are derived from hJBMMSCs ${ }^{15,16}$. Compared with bone marrow MSCs from appendicular bones, hJBMMSCs have been shown to have distinct characteristics in terms of differentiation traits ${ }^{12}$. hJBMMSCs have an increased proliferation rate and bone-forming capacity in vitro and in vivo, but less chondrogenic and adipogenic 


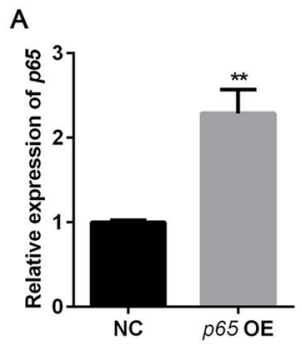

C

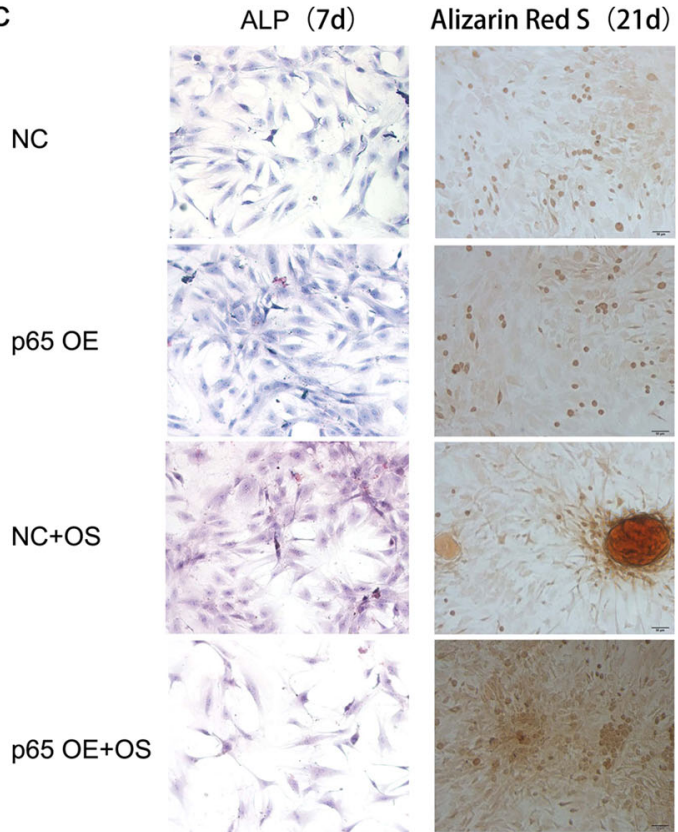

$\mathrm{F}$

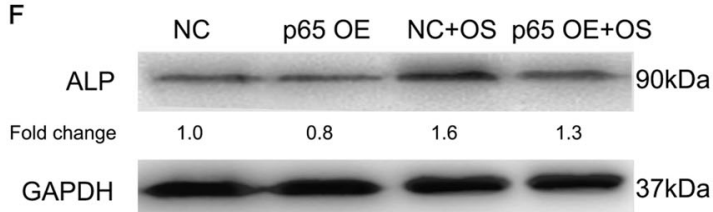

B

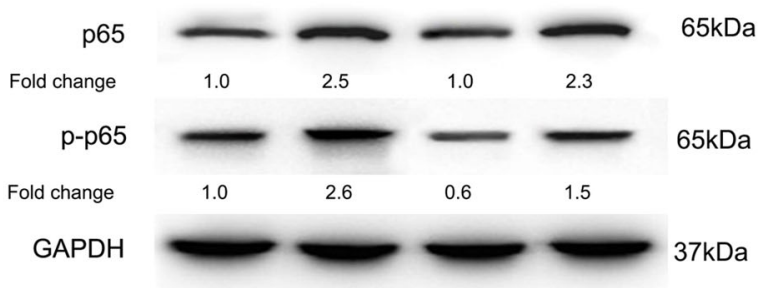

NC p65 OE NC+OS p65 OE+OS

$\mathrm{kDa}$
$\mathrm{kDa}$
$\mathrm{kDa}$
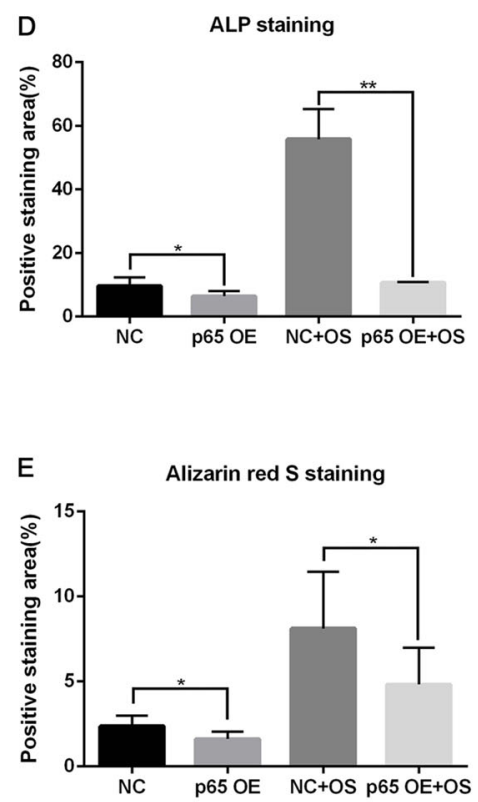

G
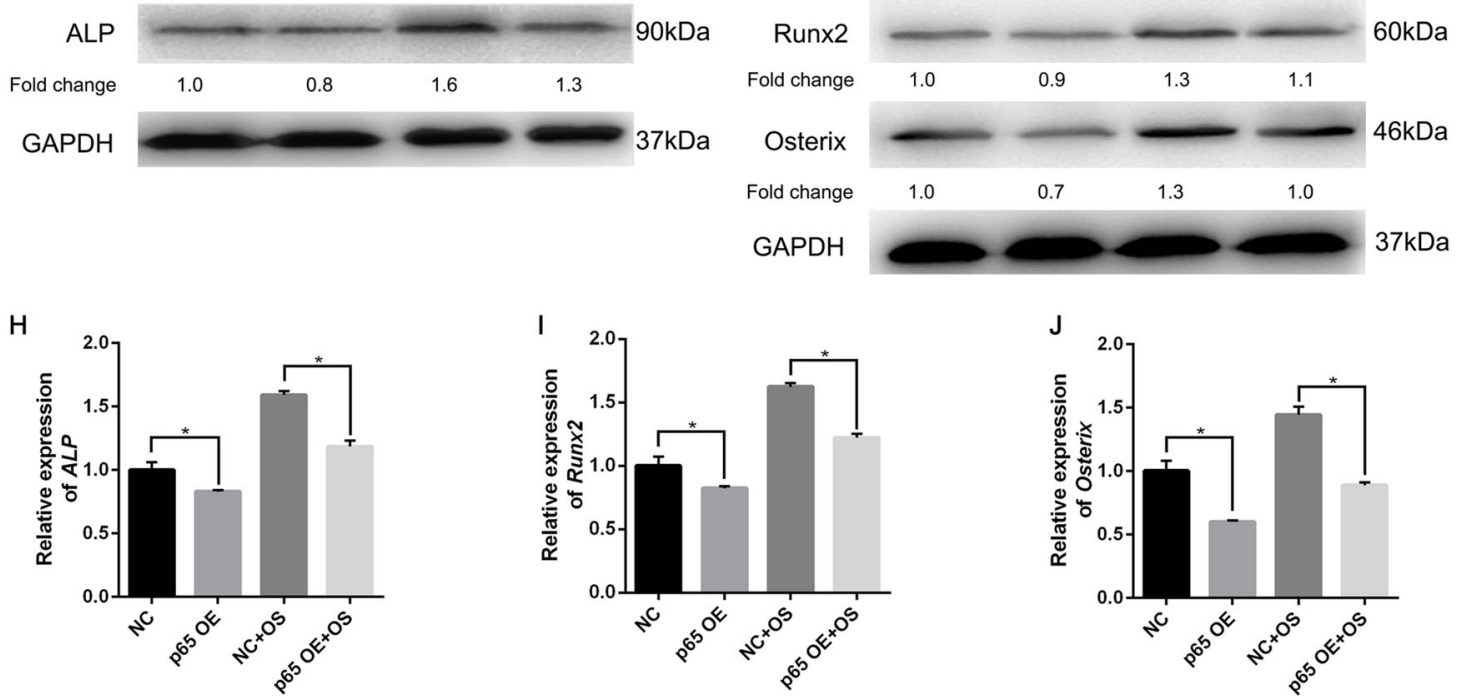

Fig. 3 Constantly activating NF-KB blocks OS-induced osteogenic differentiation of hJBMMSCs. a mRNA expression of P65 in hJBMMSCs that transfected with control vector (NC) or P65 overexpression plasmid (p65 OE). b Protein level of P65 and p-P65 in hJBMMSCs transfected with control vector or p65 overexpression plasmid, cultured in regular medium or OS. c ALP staining (7 days) and Alizarin red staining (21 days) of hJBMMSCs after cultured in regular medium or OS. $\mathbf{d}-\mathbf{e}$ Quantification of ALP staining and Alizarin red staining. $\mathbf{f}-\mathbf{g}$ Western blotting of ALP, Runx2, and Osterix in hJBMMSCs. $\mathbf{h}-\mathbf{j}$ mRNA expression of ALP, Runx2, and Osterix in hJBMMSCs $\left(n=6-10 /\right.$ group; $\left.{ }^{*} p<0.05,{ }^{* *} p<0.01\right)$ 


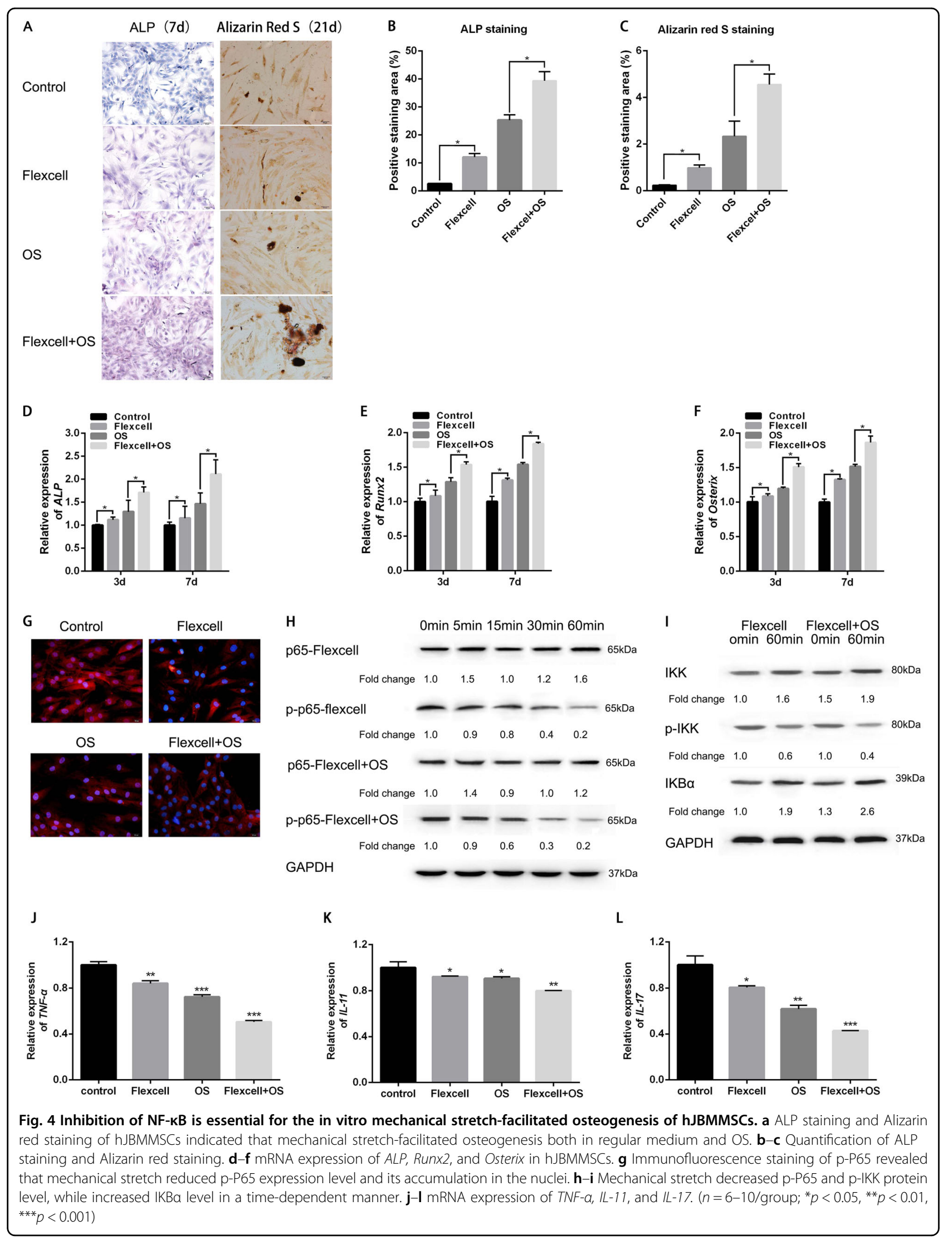




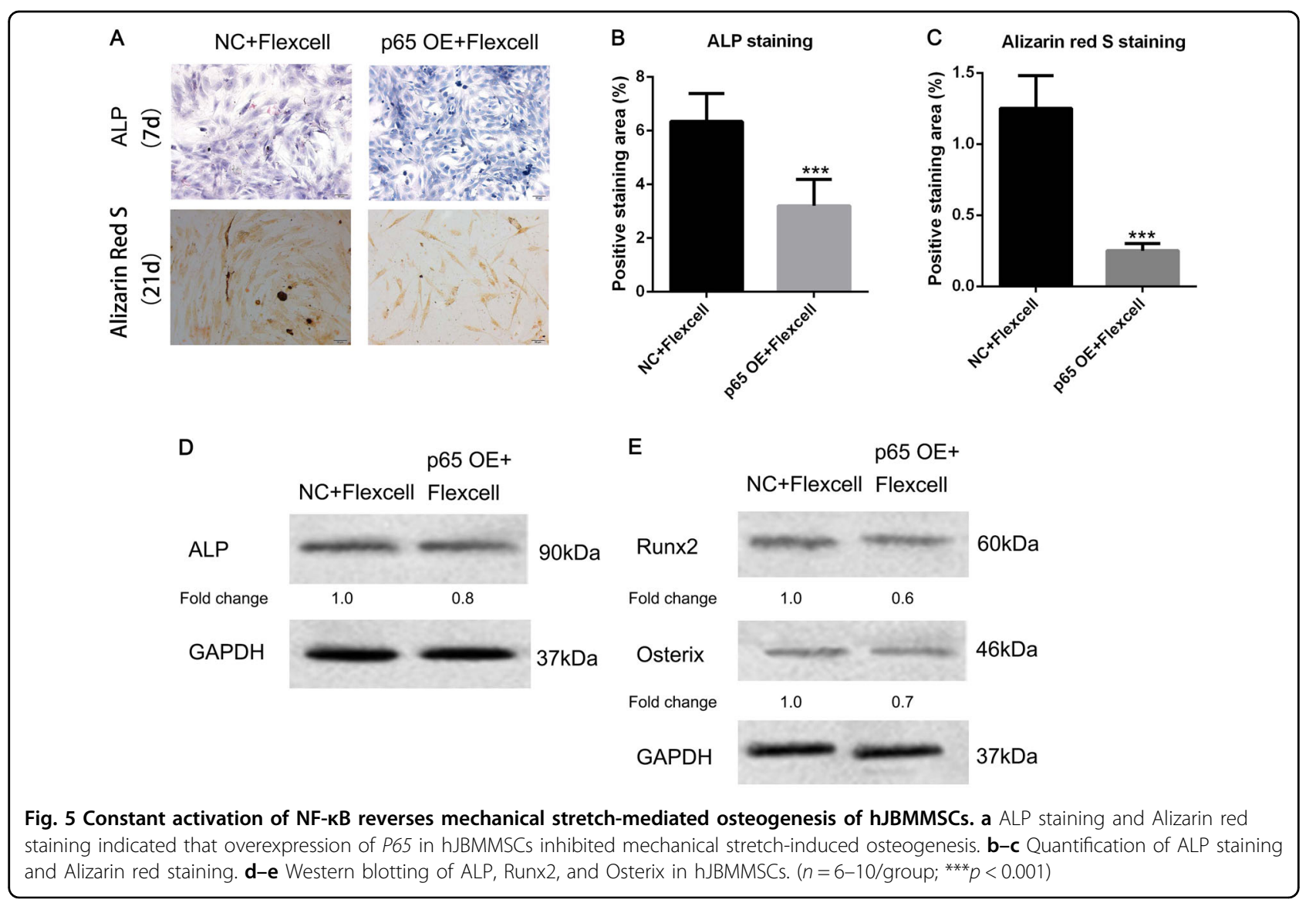

potential compared with BMMSCs from long bones ${ }^{16}$. Unlike craniofacial MSCs, which are widely studied in plasticity and regeneration ${ }^{17}$, the differentiation of hJBMMSCs has been mainly discussed under inflammatory environments, such as periodontitis, wounds, and abscesses ${ }^{18,19}$. Inflammation usually impairs multipotency of hJBMMSCs and results in weakened bone regeneration ${ }^{4}$. Nevertheless, how non-inflammatory stress, like mechanical stress, affects the behaviors of hJBMMSCs is poorly understood. In this study, we isolated hJBMMSCs from healthy humans and cultured them in vitro. Osteogenic medium and mechanical stretch both induced their osteogenic differentiation. After a mechanical stretch induction, osteoblast-specific transcription factors expression and mineralization in hJBMMSCs were increased. Since tension resulting from orthodontic forces facilitates bone regeneration, our results indicated that hJBMMSCs could be the source for osteogenesis after a mechanical stretch induction.

The Flexcell tension system was employed in our study to mimic the mechanical tension during orthodontic tooth movement ${ }^{20}$. Mechanical loading is one of the most important physical stimuli for osteoblast differentiation, for both new bone generation and maintenance ${ }^{21,22}$. Mechanical stretch on MSCs regulates Run $\times 2$ activation and favors osteoblast differentiation through the activation of the MAPK signal transduction pathways and Ras/ Raf-dependent ERK1/2 activation ${ }^{23,24}$. In mature osteoblasts, mechanical stimuli are transferred into biological responses by $\mathrm{Ca}^{2+}$ accumulation and MAPK pathway activation, such as ERK1/2 and JNK activation, which maintains the property of osteoblasts ${ }^{25,26}$. In our study, Flexcell-induced mechanical stretch-facilitated osteogenesis of hJBMMSCs by upregulation of Runx 2 and Osterix, and promoted the ALP accumulation and mineralization. Furthermore, mechanical stretch-induced osteogenesis was time-dependent. These results confirmed that bone regeneration on the tension side of orthodontic tooth movement could result from the osteogenesis of hJBMMSCs.

$\mathrm{NF}-\mathrm{\kappa B}$ is considered to inhibit the osteogenic pathway in MSCs both in vivo and in vitro ${ }^{4,10}$. NF- $\mathrm{kB}$ inhibits osteogenesis of MSCs by promoting $\beta$-catenin degradation in inflammatory microenvironments ${ }^{10}$. Even though some earlier studies showed that NF- $\mathrm{kB}$ activation by RANKL or mechanical loading promoted osteogenic differentiation of $\mathrm{MSCs}^{6,27}$, our results revealed that both osteogenic differentiation medium and mechanical stretch blocked NF- $\mathrm{KB}$ activity. Deactivation of P65, accumulation of IKB $\alpha$, and decreases of phosphorylated IKK in 
hJBMMSCs were observed during the induced osteogenesis. Meanwhile, constantly activated NF- $\mathrm{KB}$ via overexpression of P65 in hJBMMSCs efficiently blocked the OS and mechanical stretch-mediated osteogenic differentiation. Our results revealed that the mechanical stretch in non-inflammatory environments facilitates osteogenesis by inhibiting NF- $\mathrm{KB}$ activity.

In summary, our study suggested that the mechanical stretch in non-inflammatory environments facilitated osteogenesis of hJBMMSCs by blocking NF- $\mathrm{kB}$ activity. Constantly activating NF- $\mathrm{KB}$ could diminish osteoblast generation from hJBMMSCs and delay new bone generation. Our study revealed the critical role of NF- $\mathrm{KB}$ in regulation of bone generation during orthodontic tooth movement. This finding could lead to novel orthodontic appliances and improved jaw-related plasticity.

\section{Methods}

\section{Isolation of hJBMMSCs and culture}

hJBMMSCs were obtained from healthy adult human donors (aged 18-35 years) undergoing orthognathic surgery. Jaw bone debris was rinsed with $\alpha$-minimum essential medium ( $\alpha$-MEM, Gibco BRL, MD, USA) containing $100 \mathrm{U} / \mathrm{mL}$ of penicillin and $100 \mathrm{mg} / \mathrm{mL}$ of streptomycin (Invitrogen, Carlsbad, CA, USA) three times. The debris was cut into $1 \mathrm{~mm}^{3}$ pieces and passed through a 70-mm strainer to obtain single-cell suspensions. Both the cell suspensions and jaw bone pieces were seeded into 10$\mathrm{cm}$ dishes and cultured with $\alpha$-MEM supplemented with $15 \%$ fetal bovine serum (FBS, Gibco BRL) and incubated in $5 \% \mathrm{CO}_{2}$ at $37^{\circ} \mathrm{C}$. hJBMMSCs at passage three were used for the following experiments. NF-кB P65 plasmids (Santa Cruz, CA, USA) were transfected into hJBMMSCs to overexpress $P 65$.

\section{Induction of osteogenic differentiation}

A total of $2 \times 10^{5}$ cells were plated onto 6 -well plate and cultured with $\alpha$-MEM. When cell confluence reached $80 \%$, the culture medium was switched to osteogenic medium ( $\alpha$-MEM containing $100 \mathrm{nM}$ dexamethasone, 50 $\mathrm{mg} / \mathrm{mL}$ ascorbic acid, and $5 \mathrm{mM} \quad \beta$-glycerophosphate (Sigma, MO, USA)) and cultured for an additional 7 to 21 days. Calcium nodule deposit was detected by $2 \%$ Alizarin red ( $\mathrm{pH}$ 4.2, Sigma). Ten percent cetylpyridinium chloride was used as Alizarin red quantification and absorbance was measured at $562 \mathrm{~nm}$.

\section{Immunofluorescence}

Cultured hJBMMSCs were stained by immunofluorescence after treatment. Primary phospho-P65 mAb (ab86299, Abcam, Cambridge, United Kingdom), CD29 mAb (303005, Biolegend, San Diego, CA) or CD13 mAb (ab7417, Abcam) were used and followed by donkey antirabbit FITC/CY3 conjugated secondary mAb (Life
Technologies, Grand Island, NY). Cells were treated with DAPI (Vector Labs), and evaluated blindly by counting labeled cells in ten HPFs (high-power fields).

\section{Flow cytometry}

Cultured hJBMMSCs were isolated and stained with primary CD29 mAb (303005, Biolegend), CD13 mAb (ab7417, Abcam), CD45 mAb (D9M8I, Cell Signaling Technology, Beverly, MA), and CD146 mAb (ab75769, Abcam), which was followed by donkey anti-rabbit FITC/ CY3 conjugated secondary mAb (Life Technologies). hJBMMSCs were then analyzed on a FACSCalibur cytometer (BD Biosciences) and identified as $\mathrm{CD} 29^{+} \mathrm{CD} 13^{+} \mathrm{CD} 45^{-} \mathrm{CD} 146^{-}$.

\section{Employment of Flexcell system for in vitro mechanical stretch}

hJBMMSCs were plated at $5 \times 10^{5}$ cells/well in a Flexcell biaxial 6-well plate overnight. The medium was replaced by fresh basal medium or OS $24 \mathrm{~h}$ before cells being subjected to sustained mechanical stretch $(12 \%$ at $0.5 \mathrm{~Hz}$ ) by the Flexcell FX-4000 tension system (Flexcell International Corp., USA). Cell layers were then collected for further analysis.

\section{Total RNA extraction and qRT-PCR}

Total RNA was extracted from in vitro culture samples using TRIzol reagent (Invitrogen, Grand Island, NY, USA). Reverse transcriptase-polymerase chain reaction was performed with $1 \mathrm{mg}$ of RNA using the PrimeScript RT reagent kit (TaKaRa, Dalian, China). Related genes were quantified by real-time PCR using the SYBR Premix Ex Taq II kit (TaKaRa) and the CFX96 Touch Real-Time PCR Detection System (Bio-rad, Hercules, CA, USA).

\section{Protein isolation and western blotting analysis}

Total proteins were extracted with lysis buffer $(10 \mathrm{mM}$ Tris-HCL, $1 \mathrm{mM}$ EDTA, 1\% sodium dodecyl sulfate, $1 \%$ Nonidet P-40, 1:100 proteinase inhibitor cocktail, $50 \mathrm{mM}$ b-glycerophosphate, and $50 \mathrm{mM}$ sodium fluoride). Aliquots of $20-60 \mathrm{mg}$ per sample were separated by $10 \%$ sodium dodecyl sulfate-polyacrylamide gel electrophoresis, transferred to the polyvinylidene fluoride membranes and blocked with $5 \%$ nonfat milk powder in PBST (PBS with $0.1 \%$ Tween). Next, they were incubated with the following primary antibodies overnight: anti-Osterix, antiRunx2, anti-ALP, anti-P65, anti-p-P65, anti-IKB $\alpha$, antiIKK, anti-p-IKK (Cell Signaling Technology). The membranes were then incubated with horseradish peroxidaseconjugated anti-mouse or anti-rabbit IgG secondary antibodies (Boster, Wuhan, China). The blots were visualized using an enhanced chemiluminescence kit (Amersham Biosciences, Piscataway, NJ, USA) according to the manufacturer's recommended instructions. 


\section{Statistical analysis}

All results are presented as mean \pm S.D. from at least three independent experiments and were analyzed by a two-tailed unpaired Student's $t$-test using the SPSS software (IBM, Armonk, NY, USA). $p$-value $<0.05$ was considered as statistically significant.

\section{Acknowledgements}

This study was supported by grants from the National Natural Science Foundation of China $(81400538,81301669,81371169$, and 31301067) and Natural Science Foundation of Zhejiang Province (Y16C070001).

\section{Author details}

'Department of Orthodontics, Affiliated Hospital of Stomatology, Medical College, Zhejiang University, Hangzhou, Zhejiang Province, China.

${ }^{2}$ Department of Liver Surgery, Ren Ji Hospital Affiliated to Shanghai Jiao Tong University School of Medicine, Shanghai, China. ${ }^{3}$ Department of Oral Surgery, Affiliated Hospital of Stomatology, Medical College, Zhejiang University, Hangzhou, Zhejiang Province, China. ${ }^{4}$ Department of Orthodontics, Affiliated Hospital of Stomatology, Kunming Medical University, Kunming, Yunnan Province, China. ${ }^{5}$ Department of Oral Implantology, Affiliated Hospital of Stomatology, Medical College, Zhejiang University, Hangzhou, Zhejiang Province, China

\section{Authors' contributions}

H.W. designed the research and revised the manuscript; X.C. and Y.L. conducted the study, collected and analyzed the data, and wrote the manuscript; W.D. and J.S. analyzed the data and revised the manuscript; S.L., Y. L., and M.W. provided study materials and collected data.

\section{Conflict of interest}

The authors declare that they have no conflict of interest.

\section{Publisher's note}

Springer Nature remains neutral with regard to jurisdictional claims in published maps and institutional affiliations.

Supplementary Information accompanies this paper at https://doi.org/ 10.1038/s41419-018-0279-5.

Received: 12 October 2017 Revised: 28 November 2017 Accepted: 27 December 2017

Published online: 12 February 2018

\section{References}

1. Alansari, S. et al. Biological principles behind accelerated tooth movement. Semin. Orthod. 21, 151-161 (2015).

2. Roberts-Harry, D. \& Sandy, J. Orthodontics. Part 11: orthodontic tooth movement. Br. Dent. J. 7, 391-394 (2004).

3. Cho, H. H. et al. NF-kB activation stimulates osteogenic differentiation of mesenchymal stem cells derived from human adipose tissue by increasing TAZ expression. J. Cell. Physiol. 1, 168-177 (2010).

4. Chen, $X$. et al. Nuclear factor-KB modulates osteogenesis of periodontal ligament stem cells through competition with $\beta$-catenin signaling in inflammatory microenvironments. Cell Death Dis. 2, e510 (2013).
5. Wang, L. et al. Involvement of p38MAPK/NF-KB signaling pathways in osteoblasts differentiation in response to mechanical stretch. Ann. Biomed. Eng. 9, 1884-1894 (2012).

6. Clohisy, J. C., Hirayama, T., Frazier, E., Han, S. K. \& Abu-Amer, Y. NF-kB signaling blockade abolishes implant particle-induced osteoclastogenesis. J. Orthop. Res. 1, 13-20 (2004).

7. Krum, S. A., Chang, J., Miranda-Carboni, G. \& Wang, C. Y. Novel functions for NFkB: inhibition of bone formation. Nat. Rev. Rheumatol. 10,607-611 (2010)

8. Chang, J. et al. NF-KB inhibits osteogenic differentiation of mesenchymal stem cells by promoting $\beta$-catenin degradation. Proc. Natl Acad. Sci. 23, 9469-9474 (2013).

9. Ghosh, S. \& Karin, M. Missing pieces in the NF-kB puzzle. Cell 2, S81-S96 (2002).

10. Chang, J. et al. Inhibition of osteoblastic bone formation by nuclear factor-kB. Nat. Med. 6, 682-689 (2009).

11. Julien, M. et al. Phosphate-dependent regulation of MGP in osteoblasts: Role of ERK1/2 and Fra-1. J. Bone Miner. Res. 11, 1856-1868 (2009).

12. Akintoye, S. O. et al. S keletal site-specific characterization of orofacial and iliac crest human bone marrow stromal cells in same individuals. Bone 6, 758-768 (2006).

13. Yamaza, T. et al. Mouse mandible contains distinctive mesenchymal stem cells. J. Dent. Res. 3, 317-324 (2011).

14. Maleki, M., Ghanbarvand, F., Behvarz, M. R., Ejtemaei, M. \& Ghadirkhomi, E. Comparison of mesenchymal stem cell markers in multiple human adult stem cells. Int. J. Stem Cells 2, 118 (2014).

15. Mensing, N. et al. Isolation and characterization of multipotent mesenchymal stromal cells from the gingiva and the periodontal ligament of the horse. BMC Vet. Res. 1, 42 (2011).

16. Matsubara, T. et al. Alveolar bone marrow as a cell source for regenerative medicine: differences between alveolar and iliac bone marrow stromal cells. J. Bone Miner. Res. 3, 399-409 (2005).

17. Miura, M. et al. Bone marrow-derived mesenchymal stem cells for regenerative medicine in craniofacial region. Oral. Dis. 6, 514-522 (2006).

18. Kim, S. H., Seo, B. M., Choung, P. H. \& Lee, Y. M. Adult stem cell therapy for periodontal disease. Int J. Stem Cells 1, 16 (2010).

19. Boink, M. A. et al. Different wound healing properties of dermis, adipose, and gingiva mesenchymal stromal cells. Wound Repair. Regen. 1, 100-109 (2016).

20. Lohberger, B. et al. Effect of cyclic mechanical stimulation on the expression of osteogenesis genes in human intraoral mesenchymal stromal and progenitor cells. BioMed. Res. Int. 2014, 189516 (2014)

21. Haasper, C. et al. Cyclic strain induces FosB and initiates osteogenic differentiation of mesenchymal cells. Exp. Toxicol. Pathol. 6, 355-363 (2008).

22. Schwarz, S. et al. Bone marrow-derived mesenchymal stem cells migrate to healthy and damaged salivary glands following stem cell infusion. Int J. Dent. Oral. Sci. 3, 154-161 (2014).

23. Kanno, T., Takahashi, T., Tsujisawa, T., Ariyoshi, W. \& Nishihara, T. Mechanical stress-mediated Runx2 activation is dependent on Ras/ERK1/2 MAPK signaling in osteoblasts. J. Cell. Biochem. 5, 1266-1277 (2007).

24. Kelly, D. J. \& Jacobs, C. R. The role of mechanical signals in regulating chondrogenesis and osteogenesis of mesenchymal stem cells. Birth Defects Res. Part C: Embryo Today: Rev. 1, 75-85 (2010).

25. Danciu, T. E., Adam, R. M., Naruse, K., Freeman, M. R. \& Hauschka, P. V. Calcium regulates the PI3K-Akt pathway in stretched osteoblasts. FEBS Lett. 1-3, 193-197 (2003)

26. Ziros, P. G. et al. The bone-specific transcriptional regulator Cbfa1 is a target of mechanical signals in osteoblastic cells. J. Biol. Chem. 26, 23934-23941 (2002).

27. Zhou, S. et al. Inhibition of mechanical stress-induced NF-kB promotes bone formation. Oral. Dis. 1, 59-64 (2013). 